\title{
Development and Validation of HPTLC Method for Simultaneous Estimation of Diosgenin and Quercetin in Fenugreek Seeds (Trigonella foenum-graceum)
}

\author{
Omi Laila, ${ }^{1,2}$ Imtiyaz Murtaza, ${ }^{1}$ M. Z. Abdin, ${ }^{2}$ S. Ahmad, \\ Nisar Ahmad Ganai, ${ }^{4}$ and Majid Jehangir ${ }^{1}$ \\ ${ }^{1}$ Biochemistry and Molecular Biotechnology Laboratory, Biochemistry Section, Division of Post Harvest Technology, SKUAST-K, \\ Shalimar Campus, Jammu and Kashmir 191121, India \\ ${ }^{2}$ Centre for Transgenic Plant Development, Department of Biotechnology, Jamia Hamdard, Hamdard Nagar, New Delhi 110062, India \\ ${ }^{3}$ Bioactive Natural Product Laboratory, Faculty of Pharmacy, Jamia Hamdard, Hamdard Nagar, New Delhi 110062, India \\ ${ }^{4}$ TSRI, Mirgund, SKUAST-K, Kashmir, India
}

Correspondence should be addressed to Imtiyaz Murtaza; imz_murtaza@hotmail.com and M. Z. Abdin; mzabdin@rediffmail.com

Received 17 November 2013; Accepted 22 December 2013; Published 10 April 2014

Academic Editors: M. A. Lacaille-Dubois, F. J. Señoráns, and X. Wu

Copyright (c) 2014 Omi Laila et al. This is an open access article distributed under the Creative Commons Attribution License, which permits unrestricted use, distribution, and reproduction in any medium, provided the original work is properly cited.

\begin{abstract}
A sensitive, fast, and reproducible high performance thin-layer chromatographic method has been developed for simultaneous analysis of diosgenin and quercetin from fenugreek seeds, using TLC aluminium plates precoated with silica gel G60F254. Among the different combinations of mobile phases used, best separation was achieved in Toluene-ethyl acetate-formic acid $(5: 4: 1, v / v / v)$. Densitometric scanning of the plates directly at $275 \mathrm{~nm}$ was used for analysis of quercetin. While as for analysis of diosgenin, plates were scanned at $450 \mathrm{~nm}$ after spraying with anisaldehyde-sulphuric acid reagent. The retardation factorvalue of diosgenin and quercetin was found to be $0.69 \pm 0.02$ and $0.57 \pm 0.02$, respectively. The method was validated for specificity, precision (intraday and interday), accuracy, and robustness. Accuracy of the method was checked by recovery study of three different levels with the average percentage recovery of $99.13 \pm 0.26$ for diosgenin and $99.63 \pm 0.34$ for quercetin, respectively. Dried fenugreek seed samples were found to contain diosgenin in the range of $0.113-0.135 \%(\mathrm{w} / \mathrm{w})$ and quercetin in the range of $0.009-0.012 \%(\mathrm{w} / \mathrm{w})$. The present method is being reported for the first time and can be used for routine quality control and quantification of these marker compounds in various plant samples, extracts, and market formulations.
\end{abstract}

\section{Introduction}

Fenugreek (Trigonella foenum-graceum Linn., Fam. Leguminosae), highly esteemed by both east and west, has a long history of being used as a medicinal herb and has been regarded as a treatment for just about every ailment known to man [1]. Right from early times, it has been extensively used in both Indian Ayurveda and Unani systems of medicines as well as traditional Chinese medicines for treatment of epilepsy, paralysis, gout, dropsy, chronic cough, diabetes, piles sinus, and lung congestion, inflammation, infection mitigation, hair treatment, breast enhancement, and aphrodisiac effects [24]. The crop species has also long been used as a galactagogue to promote lactation in weaning mothers as well as for its ability to treat wounds and sore muscles [2, 5]. With the growing need for safer drugs, such type of traditional herbal medicines have been extensively preferred to prevent and cure human diseases because of easy accessibility by the local people and most importantly low toxicity [6]. Therefore, in view of this, human use of fenugreek is expected to increase day by day. Research literature survey suggests pharmacological properties of fenugreek seed are attributed to presence of specific bioactive compounds like steroidal diosgenin, alkaloid trigonelline, flavonoid quercetin, galactomannan, and unusual amino acid 4-hydroxyisoleucine [7]. Among these above mentioned key bioactive compounds of fenugreek, diosgenin, and quercetin (Figure 1), have been reported to be of utmost importance and are known to produce desired 
<smiles>O=c1c(O)c(-c2ccc(O)c(O)c2)oc2cc(O)cc(O)c12</smiles>

(a)

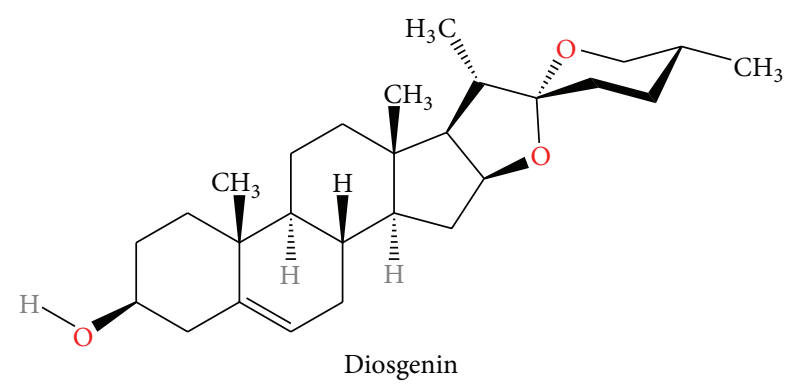

(b)

FIGURE 1: Molecular structures of quercetin and diosgenin.

effects in consumers. Quercetin, a bioflavonoid, has been reported to have anti-inflammatory, antidiabetic, and anticancer activities and other properties including improving mental and physical performance [8]. It has also been reported to attenuate thermal hyperalgesia in a mouse model of diabetic neuropathic pain [9]. Likewise, nowadays, there is considerable commercial interest in growing fenugreek for its high sapogenin content [10] mainly diosgenin (25R)-spirost5 -en-3 $\beta$-ol) that in addition to possessing anti-rheumatic and anti-viral properties, suppresses inflammation, inhibits proliferation, and induces apoptosis in a variety of tumour cells [11]. In the production of steroidal drugs and hormones such as testosterone, glucocorticoids, and progesterone, diosgenin is often used as a raw precursor [12].

Due to innumerable health benefits of such phytochemicals, currently, great attention is being given to determine quality, efficacy, and standards of the herbal raw material and formulation like Mustakarista and Mrusanivari Sura Ayurvedic Pharmacopoeia reported to contain fenugreek seeds [11]. Diosgenin and quercetin are very important bioactive constituents of fenugreek seeds; therefore, there is an urgent need to develop a simple method for simultaneous estimation of these two chemotherapeutic compounds using sophisticated instruments like high performance liquid chromatography (HPLC) and (HPTLC). Till date, several analytical methods including colorimetry, spectrophotometry, HPLC, HPTLC, and TLC have been reported for analysis of diosgenin or quercetin in fenugreek individually $[6,13$, 14]. However, literature survey shows no single quantitate method available for simultaneous estimation of diosgenin and quercetin from fenugreek or any other plant.
Keeping in view widespread use of fenugreek seeds by general population and the expected increase in its therapeutic use for a number of diseases, the present study was carried out with an objective of developing a validated sensitive and fast HPTLC method for simultaneous determination and quantification of quercetin and diosgenin in fenugreek. The method is being reported for the first time and can widely be applied for routine analysis and quality assurance of related extracts, drugs, and polyherbal formulations.

\section{Experimental}

\subsection{Plant Samples and Chemicals}

(a) Standard diosgenin (98\%) and quercetin (98\%) were procured from MP Biomedicals (USA) for development of method. Three batches of authenticated fenugreek seeds (IL1, IL2, and IL3) were procured from Division of Vegetable Sciences, Sher-e-Kashmir University of Agricultural Sciences and Technology, Shalimar, Kashmir, India.

(b) Anisaldehyde-sulfuric acid spray reagent-To $0.5 \mathrm{~mL}$ of anisaldehyde reagent (Sd fine chemicals, Mumbai, India), $10 \mathrm{~mL}$ of glacial acetic acid (Thomas Baker, Mumbai, India), and $85 \mathrm{~mL}$ methanol (Merck, India) were added followed by addition of $5 \mathrm{~mL}$ concentrated sulphuric acid (Loba Chemicals, India).

(c) All the other chemicals and solvents used in current study were of AR and HPLC grade (E. Merck, Mumbai, India).

2.2. Preparation of Standard Solution. Standard solution of diosgenin (98\%) and quercetin (98\%) were prepared either separately or in a mixture at a concentration of $1 \mathrm{mg} \mathrm{mL}^{-1}$ each in absolute methanol. Ultra sonication of mixture was required to ensure complete dissolution.

2.3. Sample Preparation. Accurately weighed fenugreek seed powder $(1.0 \mathrm{~g})$ from three batches of fenugreek was taken separately in $100 \mathrm{~mL}$ round bottom flasks. Selection of extraction solvent was performed with $25 \mathrm{~mL}$ of $1 \mathrm{M}$ acidified (sulphuric acid) ethanol $(25 \%, 50 \%, 70 \%$, and $100 \%), 1 \mathrm{M}$ acidified (sulphuric acid) methanol (50\% and 100\%), and water. The mixtures were refluxed for 60 minutes at $100^{\circ} \mathrm{C}$ in a heating block (Multi-Block, Labline Instruments, IL) to hydrolyze naturally occurring glycosylated dioscin and quercetin aglycone conjugates and to yield free diosgenin and quercetin in solution as reported earlier for these two compounds individually [15-17]. After hydrolysis, the solutions were cooled, filtered, and concentrated at $50^{\circ} \mathrm{C}$ with a rotatory evaporator (Buchi Rotavapor-R, Shivaki, India). The concentrates were made alkaline with $25 \%$ ammonia solution $(\mathrm{pH}>12)$ and diluted with double volume of distilled water. Reextraction of concentrated solutions was performed three times each with different solvents including dichloromethane (DCM), ethyl acetate, acetone, and hexane in order to get maximum yield of diosgenin and quercetin in selected solvent. The pooled extracts from each solvent were 
TABLE 1: Method validation parameters for the quantification of diosgenin and quercetin by proposed HPTLC method.

\begin{tabular}{lcc}
\hline Validation parameters & Results for diosgenin & Results for quercetin \\
\hline Regression equation & $Y=3416+27.27 X$ & $Y=1055+10.81 X$ \\
Correlation coefficient $n=3$ & $\mathbf{0 . 9 9 0}$ & 0.985 \\
Linearity range (ng/spot), $n=3$ & $100-500$ & $100-1000$ \\
$R_{f}$ & $0.69 \pm 0.02$ & $0.57 \pm 0.02$ \\
Limit of detection (ng/spot), $n=3$ & 12.52 & 15.3 \\
Limit of quantification (ng/spot), $n=3$ & 45 & 40 \\
Instrumental precision (RSD), $n=6$ & 0.42 & 0.56 \\
Method precision (RSD) $n=6$ & 0.61 & 0.67 \\
Specificity & Specific & Specific \\
Robustness (RSD), $n=3$ & 0.83 & 0.94 \\
\hline
\end{tabular}

washed first with $2 \mathrm{~mL}$ of $0.1 \mathrm{M}$ sodium hydroxide to remove the free fatty acids, and then $1 \mathrm{~mL}$ of distilled water was added to remove the remaining hydrophilic contaminants. The washed extracts were quantitatively transferred to a $50 \mathrm{~mL}$ flask, and then evaporated to dryness with a rotary evaporator (Buchi Rotavapor-R, Shivaki, India) at $50^{\circ} \mathrm{C}$. The dry residue obtained were dissolved in $5 \mathrm{~mL}$ of methanol and an aliquot of the reconstituted solution was filtered through $0.45 \mu \mathrm{m}$ polypropylene membrane filter (Fisher Scientific, India) before HPTLC analysis.

\subsection{HPTLC Instrumentation and Experimental Conditions.} Before use, precoated silica gel TLC (E. Merck, Darmstadt, Germany) plates $60 \mathrm{~F}_{254}(20 \mathrm{~cm} \times 10 \mathrm{~cm}$ with $0.2 \mathrm{~mm}$ thickness) were prewashed by dipping the plates in methanol and the solvent was allowed to overrun the plate followed by drying in fume hood. The plates were activated at $60^{\circ} \mathrm{C}$ for $5 \mathrm{~min}$ prior to chromatography. Sample solutions were applied onto the plates with semiautomatic TLC sampler Linomat V (Camag, Muttenz, Switzerland) controlled by WinCATS software version 1.4.4. A constant application rate of $80 \mathrm{nLs}^{-1}$ was employed with a band width of $3.0 \mathrm{~mm}$ and distance between two bands was $6.2 \mathrm{~mm}$. The plates were developed in $20 \times 10 \mathrm{~cm}$ twin trough glass chamber (Camag, Muttenz, Switzerland) containing $25 \mathrm{~mL}$ of mobile phase as mixture of toluene : ethyl acetate : formic acid $(5: 4: 1, v / v / v)$. The optimized chamber saturation time for mobile phase was 15 minutes at room temperature $\left(25 \pm 2^{\circ} \mathrm{C}\right)$ and 60 $\pm 5 \%$ relative humidity. The length of chromatogram run was up to $80 \mathrm{~mm}$ from the point of application $(10 \mathrm{~mm})$. After development, chromatographic plates were dried for 5 minutes in a current of air with the help of a hair dryer in normal mode. Quantitative evaluation of plate was performed with slit dimension of $3.0 \times 0.45 \mathrm{~mm}$ and scanning speed of $20 \mathrm{~mm} \mathrm{~s}^{-1}$. For quantification of quercetin, the plates were directly scanned within 10 minutes using densitometric scanner III with WinCATS software (Camag) in the UV mode with the deuterium source set at $275 \mathrm{~nm}$. The spots corresponding to quercetin were observed at $R_{f}=0.57 \pm$ 0.02 . For diosgenin, the dried plates were dipped into the anisaldehyde-sulphuric acid detection reagent and dried for 10 minutes under hot air followed by placing in an oven at $105^{\circ} \mathrm{C}$ for 10 minutes. The plates were scanned within 10 minutes using densitometric scanner III with WinCATS software (Camag) in the visible mode with the tungsten halogen source set at $450 \mathrm{~nm}$. The spots corresponding to diosgenin were observed at $R_{f}=0.69 \pm 0.02$. The data obtained was analysed by WinCATS software measuring peak areas and linear regression (Table 1).

2.5. Estimation of Diosgenin and Quercetin for Preparation of Calibration Curves. Calibration was performed by application of variable concentrations of respective standard solutions $\left(1 \mathrm{mg} \mathrm{mL}^{-1}\right)$ of quercetin and diosgenin on HPTLC plates. Both the standards were spotted at $0.1-2.0 \mu \mathrm{L}$ in triplicate on $20 \times 10 \mathrm{~cm}$ TLC plates for preparing six point linear calibration curves. The calibration graph was plotted using the concentration versus average peak area at $450 \mathrm{~nm}$ and $275 \mathrm{~nm}$ for diosgenin and quercetin, respectively. Peak area and concentration data were treated by linear leastsquares regression analysis. From each of the three samples, $4.0 \mu \mathrm{L}$ of the extracts were applied in triplicate on the HPTLC plates so that the sample zone scan areas matched the scan areas of the standards in the middle of the calibration range, that is, $500 \mathrm{ng}$ per band for quercetin and $500 \mathrm{ng}$ per band diosgenin. The experimental parameters were identical for all the above analysis.

\subsection{Validation of Method}

2.6.1. Linearity. The linearity of the method was assessed by the analysis of standard solutions $\left(1 \mathrm{mg} \mathrm{mL}^{-1}\right)$ of the two substances at six concentration levels, that is, $0.1-2.0 \mu \mathrm{L}$ of each diosgenin and quercetin standards, respectively. The linearity was evaluated using linear least-squares regression analysis for generation of calibration curve. The regression equation with slope, intercept, and coefficient of correlation ( $r$ ) was calculated (Table 1).

2.6.2. Limit of Detection (LOD) and Limit of Quantification (LOQ). The compounds were detected on the basis of $R_{f}$ value as well as UV/Visible spectral overlaying of respective standard compounds. Standards were diluted and applied on HPTLC plates to plot the calibration curves. The LOD was 
TABLE 2: Results from study of recovery for diosgenin and quercetin.

\begin{tabular}{lccccc}
\hline Compound & $\begin{array}{c}\text { Amount of } \\
\text { compound present } \\
\text { in plant material } \\
(\mu \mathrm{g} / \mathrm{g})\end{array}$ & $\begin{array}{c}\text { Amount of } \\
\text { standard added } \\
(\mathrm{ng})\end{array}$ & $\begin{array}{c}\text { Amount of } \\
\text { standard found in } \\
\text { mixture }(\mu \mathrm{g})\end{array}$ & Recovery $^{\#}$ & Average recovery $^{\#}(\%)$ \\
\hline \multirow{3}{*}{ Quercetin } & 354 & 391.3 & $742.5 \pm 0.62$ & $99.62 \pm 0.60$ & $99.55 \pm 0.59$ \\
\hline \multirow{3}{*}{ Diosgenin } & 354 & 568.8 & $918.7 \pm 0.17$ & $99.73 \pm 0.48$ & $99.63 \pm 0.34$ \\
& 354 & 643.4 & $994.8 \pm 0.48$ & $98.88 \pm 0.33$ \\
\hline
\end{tabular}

${ }^{\#}$ Mean \pm relative standard deviation (\%); $n=3$.

determined based on the lowest concentration detected by the instrument from each of two standards, while the LOQ was determined based on the lowest concentration quantified in the samples (Table 1).

2.6.3. System Precision. Instrumental precision was checked by analysing spots derived from applying $250 \mathrm{ng}$ of standard diosgenin per spot and $500 \mathrm{ng}$ of standard quercetin per spot six times each, respectively. The precision of $R_{f}$ values were evaluated and expressed as percentage relative standard deviation (RSD, \%,) for the specific marker band (Table 1).

2.6.4. Method Precision (Repeatability). The repeatability of the method was assessed by extracting test portions of each of the three seed samples 6 times each and analysing each extract accordingly by HPTLC method. The percentage relative standard deviations were expressed as RSD (\%) (Table 1).

2.6.5. Accuracy (Recovery). The accuracy of the method was assessed by spiking preanalyzed samples with known amounts of standard quercetin and diosgenin solution and then reanalysed by the HPTLC method. The spiking was done at three different concentration levels and average percent recovery at each concentration levels was calculated (Table 2). The experiment was conducted in triplicate.

2.6.6. Intermediate Precision (Reproducibility). The intraassay precision of the developed methodology was evaluated by analysing three replicates of two substances at three concentration levels, that is, standard solutions of quercetin (200, 400, and $900 \mathrm{ng}$ per band) and diosgenin (100, 250, and $450 \mathrm{ng}$ per band) on the same day. While interassay precision was evaluated at the same levels in three different laboratory days. Further, test portions of the seed samples from the same batches were processed and analysed three times on three different laboratory days. The data generated was evaluated and results expressed as (\%) RSD (Table 3).

2.6.7. Specificity. The specificity of the method was ascertained by analysing standards and extract samples of equivalent concentrations. The bands for diosgenin and quercetin in the sample were confirmed by comparing the $R_{f}$ value and
TABle 3: Precision by the proposed HPTLC method for diosgenin and quercetin.

\begin{tabular}{lccc}
\hline Compound & $\begin{array}{c}\text { Concentration } \\
\text { (ng per band ) }\end{array}$ & \multicolumn{2}{c}{ Precision, as RSD (\%) } \\
Intraday & Interday \\
\hline \multirow{3}{*}{ Quercetin } & 200 & 0.96 & 1.10 \\
& 400 & 1.04 & 0.80 \\
Diosgenin & 900 & 0.82 & 1.06 \\
\hline & 100 & 1.27 & 0.96 \\
& 250 & 1.15 & 1.30 \\
& 450 & 1.14 & 0.96 \\
\hline
\end{tabular}

spectra of the band from sample and standard solution. The peak purity (98\%) of standard diosgenin and quercetin was assessed by comparing the spectra at three levels including peak start, peak apex, and peak end. Peak purity of the sample was determined by comparing overlaid spectra of the diosgenin or quercetin in sample and standard chromatograms (Figures 3(a), 3(b), and 6).

2.6.8. Robustness. The robustness of the method was studied by introducing small deliberate changes in experimental conditions and results examined. For example, change in composition of mobile phase (Toluene-ethyl acetate-formic acid) was tried at four levels, that is, $5: 4: 1,5: 3: 1,4: 4: 1$, and $4: 5: 1 v / v / v$ as well as effect of amount of mobile phase, time from spotting to chromatography, development distance $( \pm 5 \mathrm{~mm})$, and scanning were also investigated. The robustness of the method was performed in triplicate and the results were expressed as RSD (\%) of the peak area (Table 1).

\section{Results and Discussion}

A quantative HPTLC method that gives dense and compact spots with significant $R_{f}$ values for simultaneous determination of diosgenin and quercetin in fenugreek seeds was developed. The effect of extraction solvent was investigated by introducing a single factor test. As the flavonoids and saponins are polar compounds, the polar solvents like ethanol, methanol, water, and their combination can be employed for better extraction of these substances than 


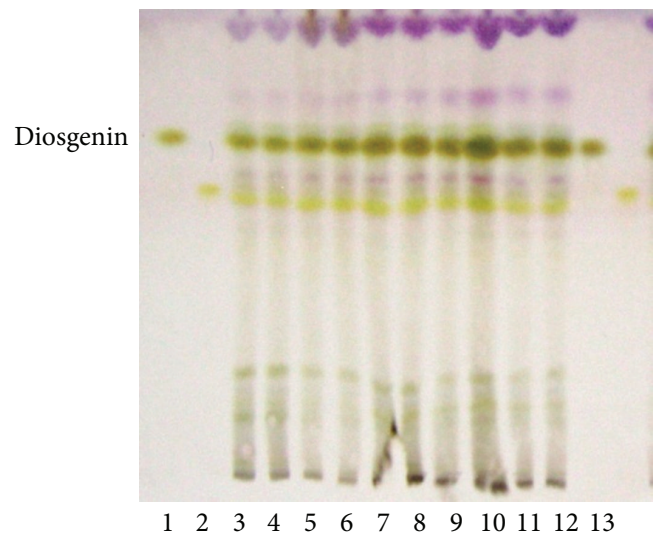

(a)

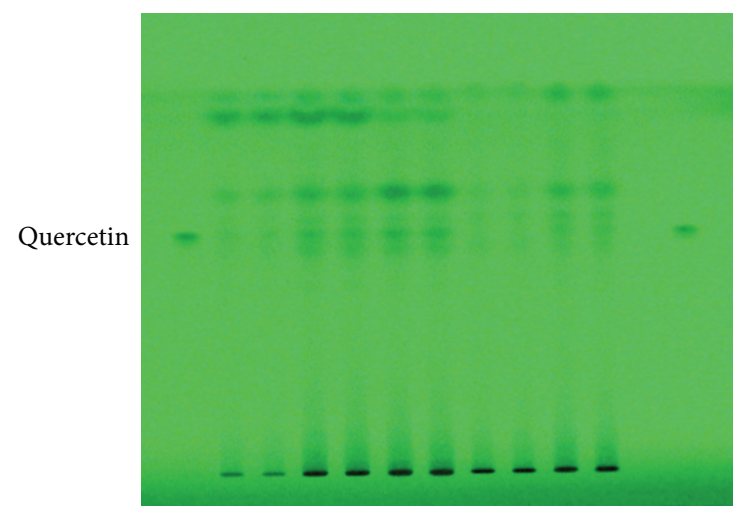

$\begin{array}{lllllllllllll}14 & 15 & 16 & 17 & 18 & 19 & 20 & 21 & 22 & 23 & 24 & 25 & 26\end{array}$

(b)

FIGURE 2: Images of HPTLC separation of extracts of samples of fenugreek seeds. Tracks 1 and 13 represent diosgenin standard (500 ng per band) and 3-12 represent diosgenin from IL1, IL2, and IL3 seed cultivars. Tracks 14 and 26 represent quercetin standard (500 ng per band) and 15-24 represent quercetin from IL1, IL2, and IL3 seed cultivars. The mobile phase was Toluene-ethyl acetate formic acid $(5: 4: 1, v / v / v)$

nonpolar ones. For development of successful method, the first important step is to optimise the solvent system for good extraction efficiency. In the current study, three solvent systems namely ethanol $(25,50,70$, and $100 \%)$, methanol $(50 \%$, $100 \%)$, and water were investigated. Small amount of acid or water in the hydrolysing solvent can significantly improve the hydrolytic efficiency due to the increased polarity of the solvent. As shown in Figure 4, acidified absolute ethanol (100\%) was found to be the optimum extraction solvent for both quercetin and diosgenin as acidification enables the solvent to penetrate the plant matrix easily and facilitate better mass transfer of saponins and flavonoids into the extracting solvent. To optimise the efficient reextraction of diosgenin and quercetin from acidified ethanol extracts, different solvent including n-hexane, acetone, dichloromethane, and ethyl acetate were evaluated. Among different solvents tested, dichloromethane was found to be the optimal reextraction solvent (Figure 5).
As standardization of suitable mobile phase equally plays a very crucial role in chromatographic methods.As far as, individual estimation of diosgenin and quercetin by chromatographic methods is concerned, a number of solvent systems have been reported. However, there has not been cited a single report for separation of diosgenin and quercetin simultaneously in a single solvent system. Therefore, in this study, several solvent systems used for individual estimation of quercetin and diosgenin were investigated to evaluate the combinatorial separation of these two compounds in a single solvent system and between different components of the extract. Among the different solvents systems investigated, mobile phase consisting of toluene : ethyl acetate : formic acid in the ratio of $5: 4: 1 v / v / v$ demonstrated compact spots (Figure 2) with typical Gaussian shaped peaks for diosgenin $\left(R_{f}=0.69 \pm 0.02\right)$ and quercetin $\left(R_{f}=0.57 \pm 0.02\right)$ with good resolution between other peaks of the extract (Figures 3(a) and 3(b)). The six point linear calibration curves of quercetin and diosgenin were linear in the range of 100-1000 ng for quercetin and 100-500 ng for diosgenin, which indicates that the method has good sensitivity. Both the compounds were separated clearly and acted as reference for identification of them in sample extracts from IL1, IL2, and IL3. The identity of bands of quercetin, and diosgenin in sample extracts were confirmed by overlaying their UV-Vis absorption spectrum with that of standards using a CAMAG TLC scanner 3, version 1.2.3 (Figure 4). Specificity of the proposed method was evaluated by comparing the $R_{f}$ values acquired from standards and from test samples separated under identical conditions (Figures 3 and 4). The purity (98\%) of the bands of diosgenin and quercetin in sample extracts was confirmed by comparing the spectra at various points including peak start, peak apex, and peak end positions of the spot. Good correlation $(r=0.99)$ was obtained between overlaid spectra of diosgenin and quercetin in standard and sample extracts (Figure 6).

As shown in Table 1, the lower limits of detection (LOD) obtained for quercetin and diosgenin were 15.3 and $12.52 \mathrm{ng} / \mathrm{spot}$, respectively, with good linearities, while the limit of quantification (LOQ) obtained was 45 and $40 \mathrm{ng} \mathrm{spot}^{-1}$, respectively, indicating the sensitivity of the method to be adequate. The instrumental precision and repeatability of the method were assessed by determination of the RSD (\%) of peak area (Table 1). Intraday and interday precision were studied by triplicate assay of three different concentrations of diosgenin (100, 250, and $450 \mathrm{ng}$ per band $\mathrm{ng} / \mathrm{band}$ ) and quercetin (200, 400, and $900 \mathrm{ng} / \mathrm{band})$ on the same day and on three different days. Low RSD values obtained indicated that the method is precise (Table 2). As shown in Table 3, good recoveries were obtained by the enrichment of the sample at three different concentration levels. The percent recoveries obtained after sample processing and applying were found to be encouraging that lie in the range of $99.13 \pm 0.26 \%$ for diosgenin and $99.63 \pm 0.34 \%$ for quercetin. Measurements of parameters for determination of robustness indicated that the method was able to withstand minor experimental changes (Table 1).

Interestingly, while using the developed method, the amount of diosgenin in dried powdered samples of fenugreek 

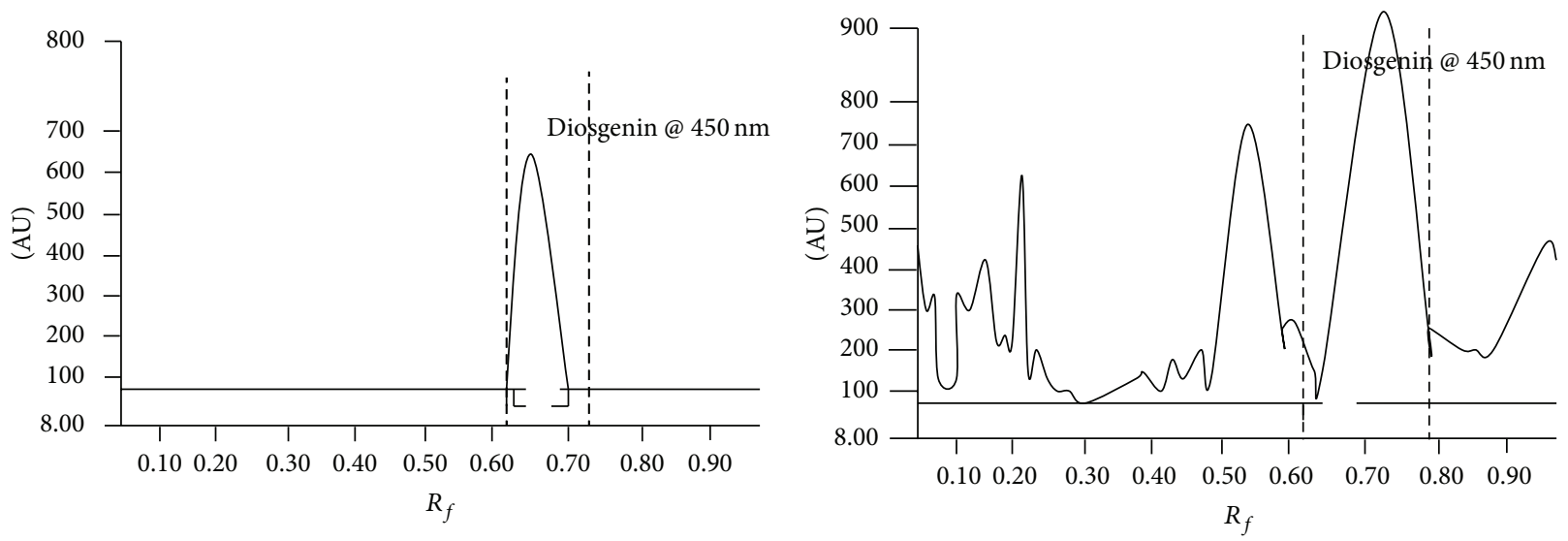

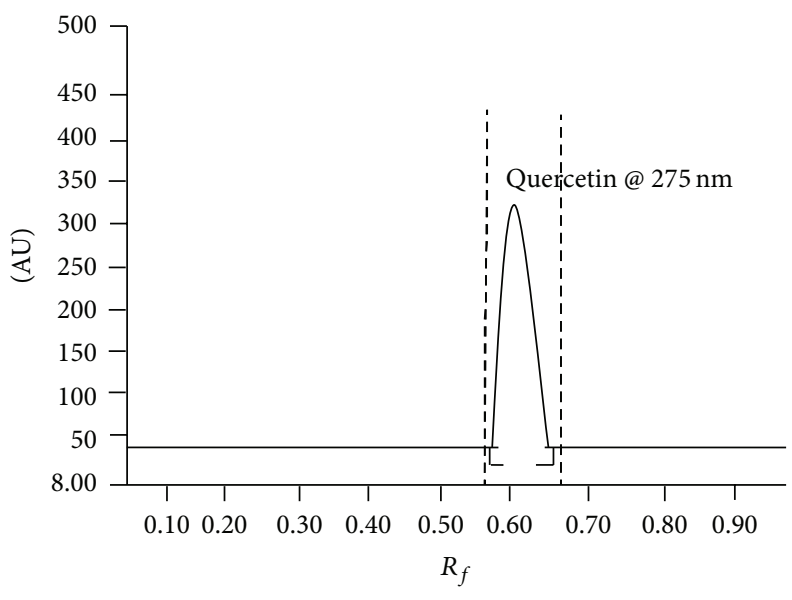

(a)

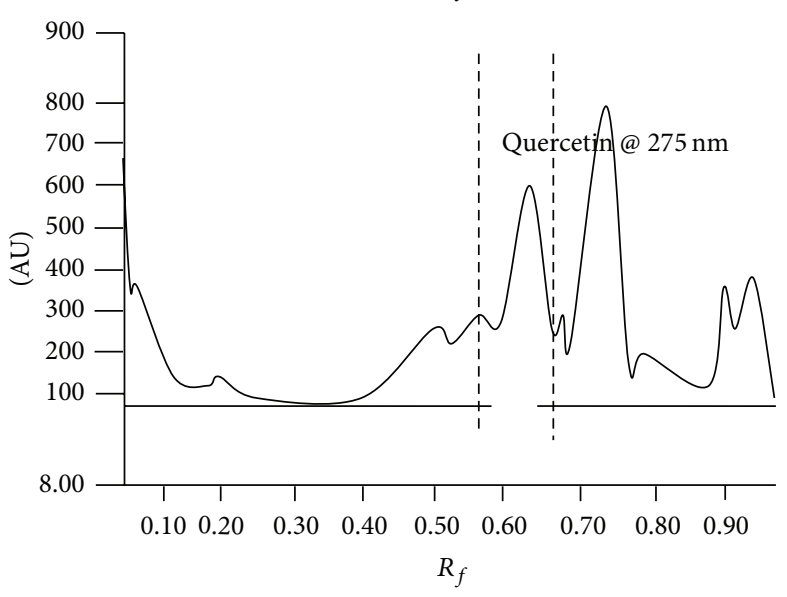

(b)

FIGURE 3: (a) Chromatogram profile of standard diosgenin and quercetin recorded at $450 \mathrm{~nm}$ and $275 \mathrm{~nm}$, respectively, by HPTLC method. (b) Chromatogram of fenugreek seed powder obtained from IL3 cultivar at $450 \mathrm{~nm}$ and $275 \mathrm{~nm}$ for estimation of diosgenin and quercetin, respectively, by the proposed method.

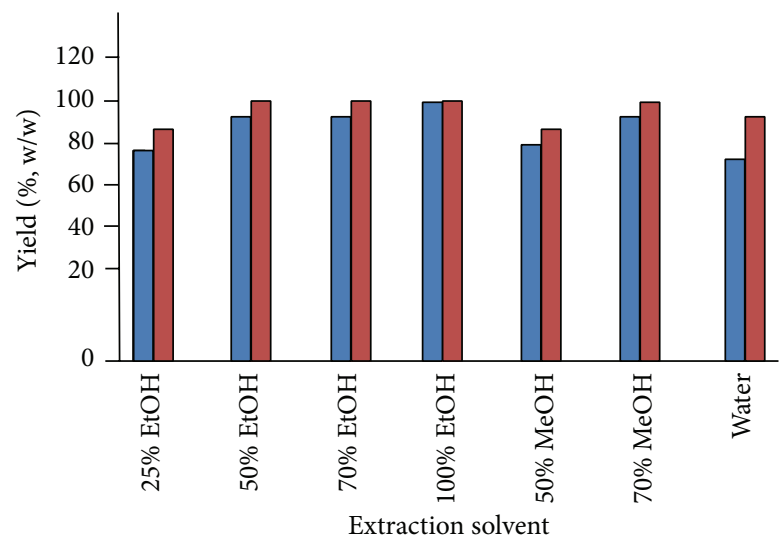

๑ Diosgenin

口 Quercetin

FIGURE 4: Effect of change in extraction solvent composition on efficiency of proposed HPTLC based simultaneous extraction of diosgenin and quercetin. 


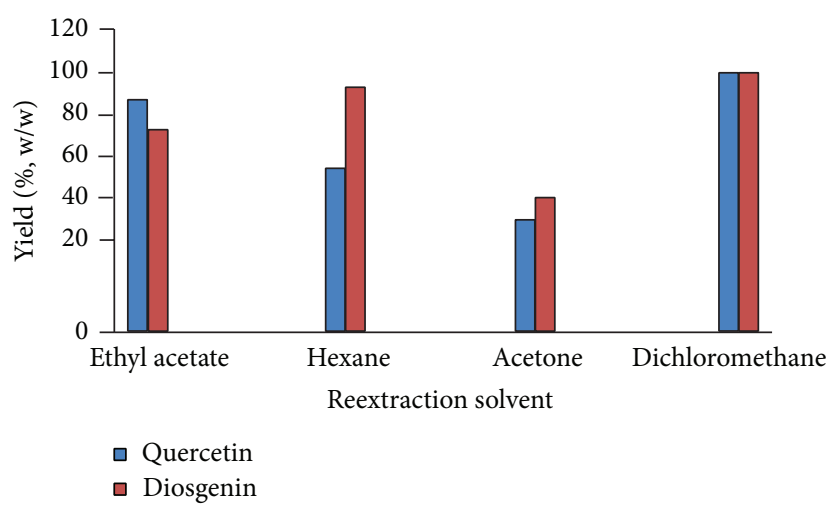

FIGURE 5: Optimisation of reextraction solvents for maximum yield of diosgenin and quercetin simultaneously.

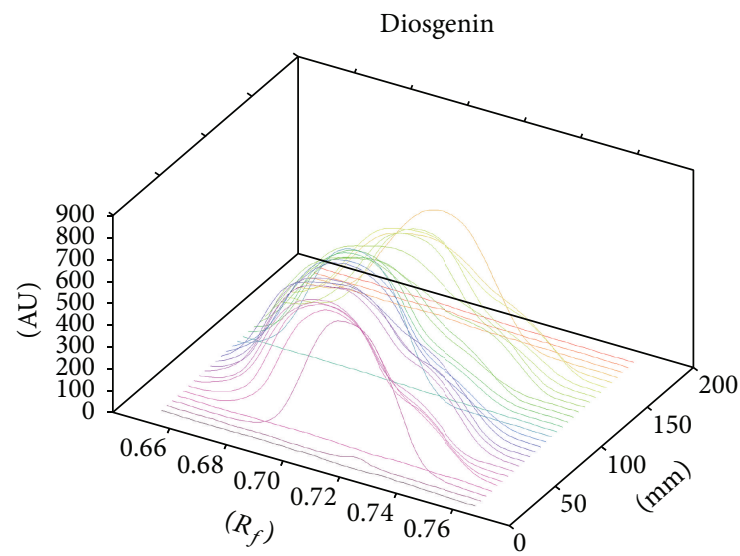

(a)

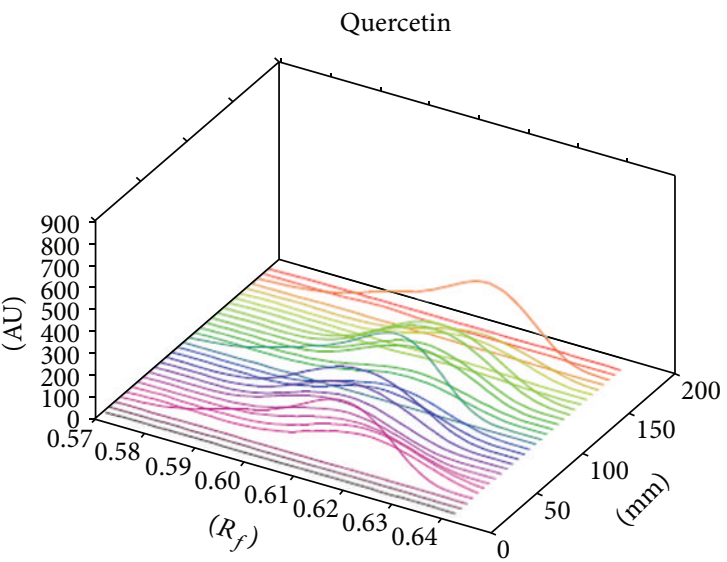

(b)

FIGURE 6: Overlaid in situ spectra of diosgenin and quercetin acquired by proposed method from standards and samples (peak purity of the sample).

seed obtained from IL1, IL2, and 1L3 cultivars was found to be $0.116 \%(\mathrm{w} / \mathrm{w}), 0.135 \%(\mathrm{w} / \mathrm{w})$, and $0.113 \%(\mathrm{w} / \mathrm{w})$, respectively, and quercetin to be $0.009 \%(\mathrm{w} / \mathrm{w})$ in IL1, $0.0121 \%$ in IL2, and $0.011 \%$ in IL3 cultivar (Table 4 ). The values are in good agreement with those values cited earlier for fenugreek in
TABLE 4: Amount of marker compounds diosgenin and quercetin quantified in samples of fenugreek seeds by proposed method.

\begin{tabular}{lcc}
\hline \multirow{2}{*}{ Samples } & \multicolumn{2}{c}{ Content $(\%, \mathrm{~W} / \mathrm{W})$} \\
& Diosgenin $(n=6)$ & Quercetin $(n=6)$ \\
\hline (1) IL1 (AGR 548) & $0.116 \pm 0.01$ & $0.009 \pm 0.001$ \\
(2) IL2 (Methi local) & $0.135 \pm 0.01$ & $0.012 \pm 0.001$ \\
(3) IL3 (Shalimar improved) & $0.113 \pm 0.01$ & $0.011 \pm 0.001$ \\
\hline
\end{tabular}

the literature and thus make this method valuable tool for accurate detection of such compounds simultaneously [18, 19].

\section{Conclusion}

TLC is an invaluable tool for standardization of herbal drugs and health foods. As herbal preparations have chemical complexities due to presence of different bioactive constituents and it is not possible to determine them by one method. The reported HPTLC method is an attractive simple, rapid, and selective method for the simultaneous quantitative determination of flavonoid quercetin and steroid diosgenin in fenugreek extract. This method could be widely applied for direct routine analysis and quality assurance of related extracts and drugs. Through this reported method, bioactive constituents of other plants with such attributes could be identified and used for selection of suitable genotypes that can be further developed into cultivars specific for the natural health product processing industry.

\section{Conflict of Interests}

The authors declare no conflict of interests.

\section{Acknowledgments}

The authors are highly thankful to UGC, New Delhi, for its financial assistance (fund no. 37-525/2009-SR) and Professor S. A. Wani, Research Director, Directorate of Research, SKUAST-K, Shalimar Campus, Kashmir, for his support. 


\section{References}

[1] G. A. Petropoulos, Fenugreek, the Genus Trigonella, Taylor and Francis, London, UK, 2002.

[2] D. Tiran, "The use of fenugreek for breast feeding women," Complementary Therapies in Nursing and Midwifery, vol. 9, no. 3, pp. 155-156, 2003.

[3] N. K. Leela and K. M. Shafeekh, "Fenugreek," in Chemistry of Spices, V. A. Parthasarathy, B. Chempakam, and T. J. Zachariah, Eds., pp. 242-259, Biddles Ltd, CAB International, King's Lynn, UK, 2008.

[4] C. L. Gopu, S. S. Gilda, A. R. Paradkar, and K. R. Mahadik, "Development and validation of a Densitometric TLC method for analysis of trigonelline and 4-hydroxyisoleucine in fenugreek seeds," Acta Chromatographica, vol. 20, no. 4, pp. 709-719, 2008.

[5] M. Rguibi and R. Belahsen, "Body size preferences and sociocultural influences on attitudes towards obesity among Moroccan Sahraoui women," Body Image, vol. 3, no. 4, pp. 395-400, 2006.

[6] A. Swaroop, A. Prakash Gupta, and A. Kumar Sinha, "Simultaneous determination of quercetin, rutin and coumaric acid in flowers of Rhododendron arboreum by HPTLC," Chromatographia, vol. 62, no. 11-12, pp. 649-652, 2005.

[7] F. A. Toppo, R. Akhand, and A. K. Pathak, "Pharmacological actions and potential uses of Trigonella foenum-graecum: a review," Asian Journal of Pharmaceutical and Clinical Research, vol. 2, no. 4, pp. 29-38, 2009.

[8] A. T. Jan, M. R. Kamli, I. Murtaza, J. B. Singh, A. Ali, and Q. M. R. Haq, "Dietary flavonoid quercetin and associated health benefits-an overview," Food Reviews International, vol. 26, no. 3, pp. 302-317, 2010.

[9] M. Anjaneyulu and K. Chopra, "Quercetin, a bioflavonoid, attenuates thermal hyperalgesia in a mouse model of diabetic neuropathic pain," Progress in Neuro-Psychopharmacology and Biological Psychiatry, vol. 27, no. 6, pp. 1001-1005, 2003.

[10] R. Randhir, Y.-T. Lin, and K. Shetty, "Phenolics, their antioxidant and antimicrobial activity in dark germinated fenugreek sprouts in response to peptide and phytochemical elicitors," Asia Pacific Journal of Clinical Nutrition, vol. 13, no. 3, pp. 295307, 2004.

[11] D. H. Nagore, P. S. Patail, and V. B. Kuber, "Comparison between high performance liquid chromatography and high performance thin layer chromatography determination of Diosgenin from fenugreek seeds," International Journal of Green Pharmacy, vol. 6, pp. 315-320, 2012.

[12] A. Mehrafarin, A. Qaderi, S. Rezazadeh, H. Naghdi Badi, G. Noormohammadi, and E. Zand, "Bioengineering of important secondary metabolites and metabolic pathways in fenugreek (Trigonella foenum-graecum L.)," Journal of Medicinal Plants, vol. 9, no. 35, pp. 1-18, 2010.

[13] V. B. Kshirsagar, U. A. Deokate, V. B. Bharkad, and S. S. Khadabadi, "HPTLC method development and validation for simultaneous estimation of diosenin and levodopa in marketed formulations," Asian Journal of Research in Chemistry, vol. 1, pp. 36-39, 2008.

[14] H. Yang, B. Chen, X. Wang et al., "Rapid quantitative analysis of diosgenin in the tubers of Dioscorea zingiberensis $\mathrm{CH}$ Wright by coupling cellulose enzymolysis and two-phase acid hydrolysis in tandem with HPLC-UV,' Natural Product Research, vol. 27, pp. 1933-1935, 2013.
[15] H. J. Shah and S. S. Lele, "Extraction of diosgenin, a bioactive compound from natural source Dioscorea alata Var purpurae," Journal of Analytical \& Bioanalytical Techniques, vol. 3, no. 4, 2012.

[16] V. B. Warke, T. A. Deshmukh, and V. R. Patil, "Development and validation of RP-HPLC method for estimation of diosgenin in pharmaceutical dosage form," Asian Journal of Pharmaceutical and Clinical Research, vol. 4, no. 1, pp. 126-128, 2011.

[17] H. P. V. Rupasinghe, P. Kathirvel, and G. M. Huber, "Ultrasonication-assisted solvent extraction of quercetin glycosides from "Idared" apple peels," Molecules, vol. 16, no. 12, pp. 9783-9791, 2011.

[18] P. D. Trivedi, K. Pundarikakshudu, S. Rathnam, and K. S. Shah, "A validated quantitative thin-layer chromatographic methoc for estimation of diosgenin in various plant samples, extract, and market formulation," Journal of AOAC International, vol. 90, no. 2, pp. 358-363, 2007.

[19] A. Dua, S. Vats, V. Singh, and R. Mahajan, "Protection of biomolecules against in vitro oxidative damage by the antioxidants from methanolic extracts of Trigonella foenum graecum seeds," International Journal of Pharmaceutical Sciences and Research, vol. 4, pp. 3080-3086, 2013. 

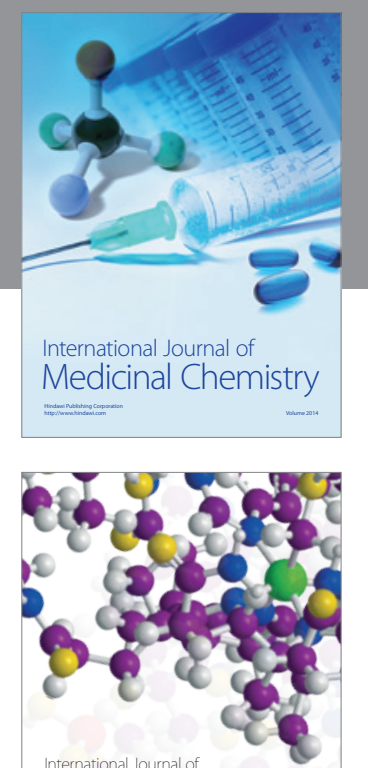

\section{Carbohydrate} Chemistry

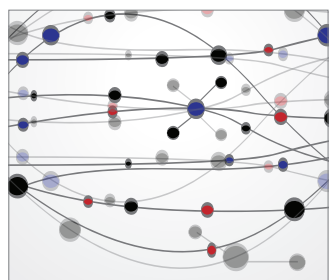

The Scientific World Journal
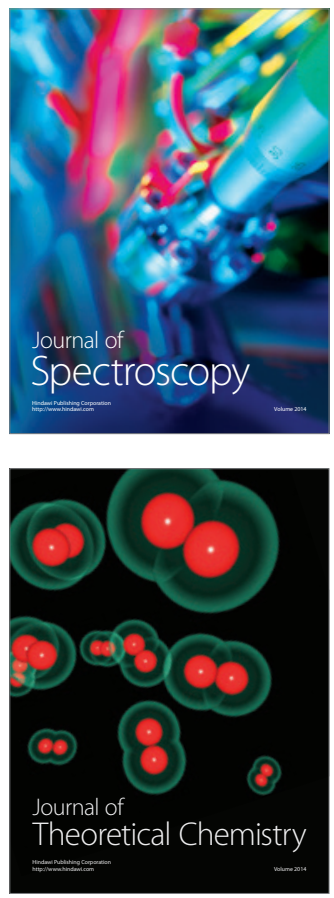
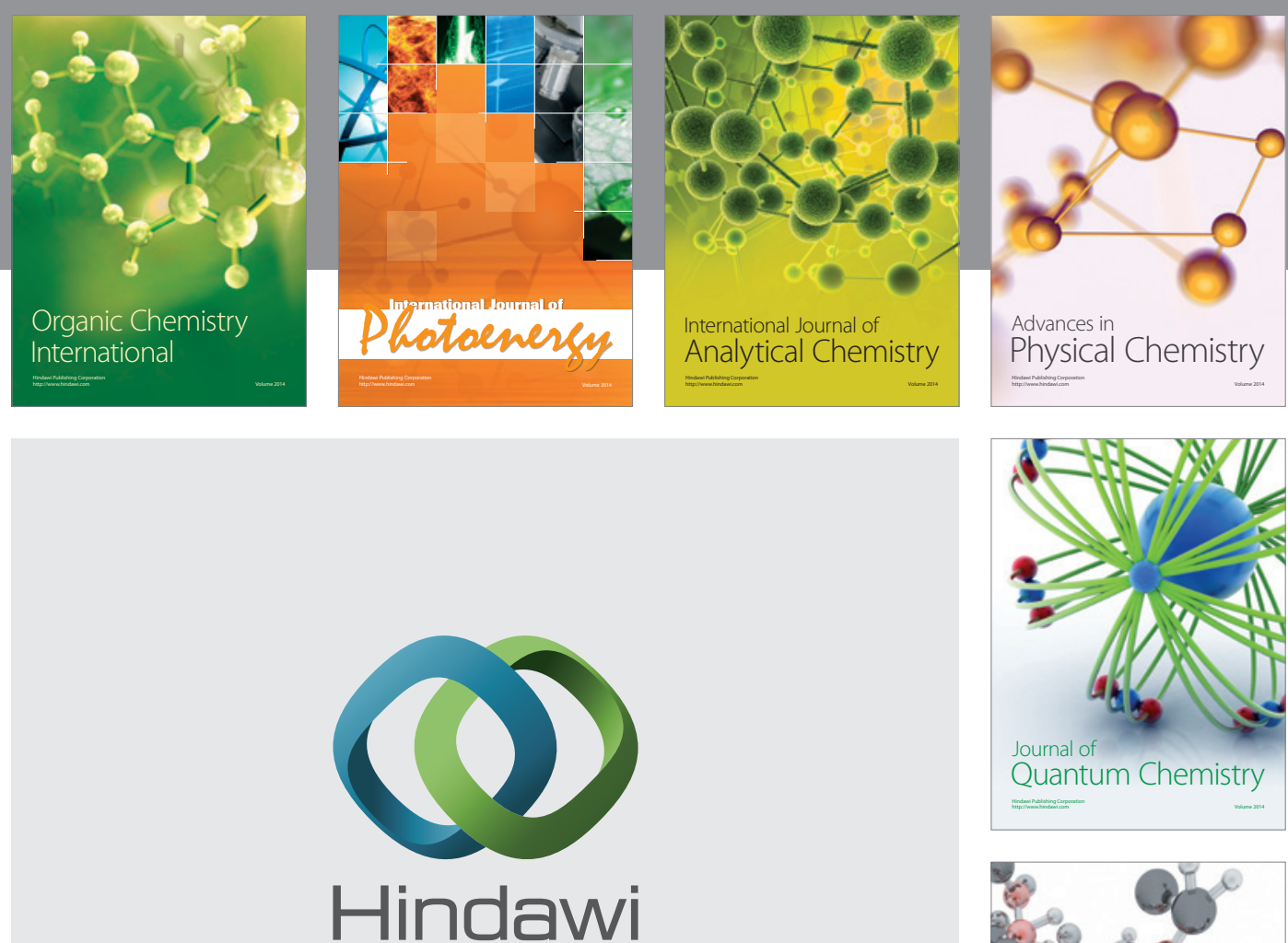

Submit your manuscripts at

http://www.hindawi.com

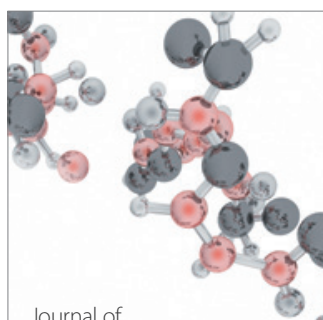

Analytical Methods

in Chemistry

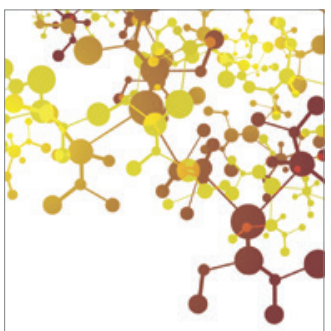

Journal of

Applied Chemistry

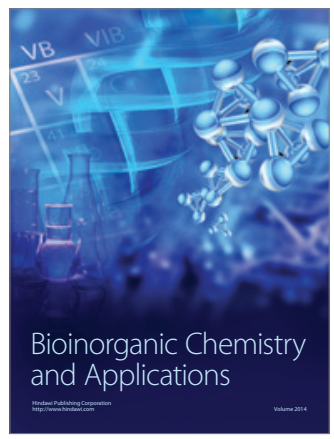

Inorganic Chemistry
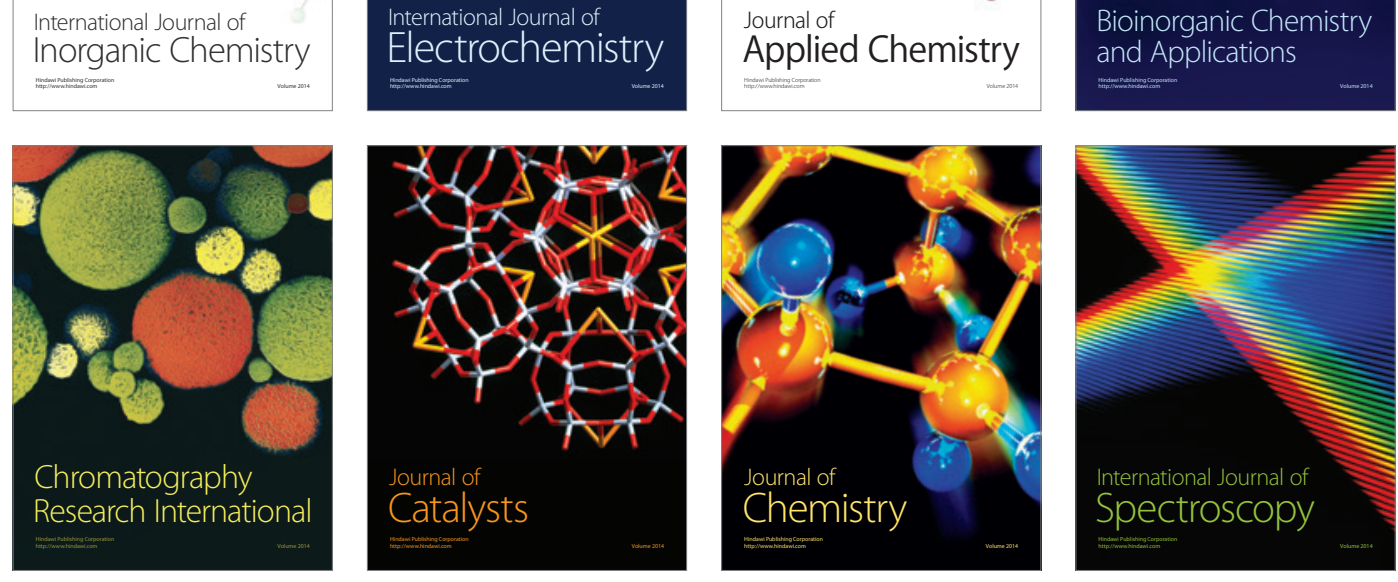